\title{
Visualizing voltage
}

Two recently developed genetically encoded voltage sensors allow scientists to image the activity of defined neuronal populations.

How brains process information is a daunting question to answer. Genetically encoded sensors help address this problem as activity in neurons of interest can be visualized. Calcium sensors, which measure activity indirectly, have been the tools of choice, but voltage sensors are slowly catching up in popularity. The groups of Michael Lin and Mark Schnitzer, both at Stanford University, have developed voltage sensors that report neural activity at high speed.

"Voltage sensors have long been on the wish list

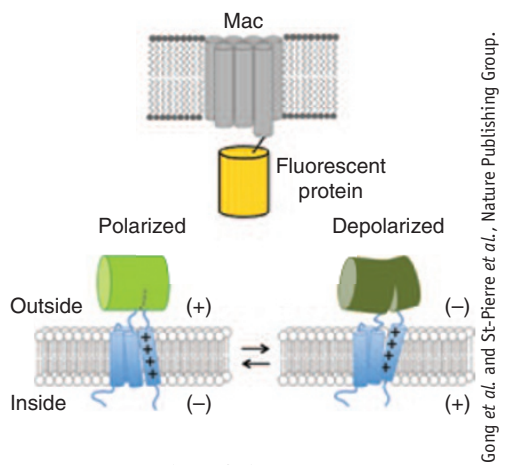

Design principles of the MacQ sensors (top) and ASAP1 (bottom). of genetically encoded sensors," says Lin. The first voltage sensor was reported in 1997. Since then, two classes of sensors with different designs have emerged. In one type, two fluorescent proteins capable of fluorescence resonance energy transfer (FRET) are coupled by a voltage-sensitive domain. Voltage-dependent conformational changes lead to changes in FRET signals. The other type is based on rhodopsins such as archaerhodopsin. In this case, changes in membrane voltage result in changes of the endogenous fluorescence of these proteins. However, these sensors have generally been either too slow to record activity from fast-spiking neurons or too dim to be used in tissues.

The voltage sensor from Yiyang Gong and the Schnitzer lab is based on the latter principle, but it also incorporates a fluorescent protein as a FRET donor (Gong et al., 2014). The researchers fused a modified rhodopsin from Leptosphaeria maculans to either mOrange 2 or mCitrine,

\section{SYNTHETIC BIOLOGY}

\section{CRISPR CIRCUITS}

CRISPR-based transcriptional activators and repressors for synthetic circuits.

When it comes to designing genetic circuits-that is, the combination of biological parts to carry out logical functions - there are several things on a synthetic biologist's wish list: easy programming, small DNA footprints and a large library of parts that can be used to build complex architectures. The clustered, regularly interspaced, short palindromic repeats (CRISPR)-Cas9 system promises to deliver on all of the above: the guide RNA (gRNA) that targets active or inactive nuclease Cas9 to its complementary DNA can be easily designed, and gRNAs are small and can be quickly created for many targets.

Two independent studies from researchers at the Massachusetts Institute of Technology recently presented CRISPR-based transcriptional cascades.

Tim Lu and his postdoctoral fellow Lior Nissim and graduate student Samuel Perli created tunable CRISPR-based transcriptional activators, with Cas 9 fused to an activation domain (Nissim et al., 2014). "With the CRISPR-Cas system we have the ability to build much more sophisticated circuit architecture," says Lu, "but previously gRNAs have been expressed from pol III promoters, and that limits you in terms of the types of circuits you can build." Therefore, they first focused on expressing the gRNA from polymerase II (pol II) promoters, which should enable inducible and tissue-specific control via synthetic or endogenous promoters.

But simply driving gRNA from a pol II promoter did not lead to functional gRNAs in their hands. The team instead developed several ways of releasing the gRNA from a longer transcript. These approaches allowed the expression of multiple gRNAs from the same transcript, important for the activation of endogenous genes. The team also showed layered transcriptional control: one gRNA-Cas9 complex triggers expression of another gRNA, which, together with Cas9, activates an output reporter. 
thereby creating MacQ-mOrange2 and MacQ-mCitrine. This strategy combines the fast kinetic properties of the rhodopsin with the brightness conferred by the fluorescent proteins.

On the other hand, Lin's ASAP1 sensor is designed from scratch (St-Pierre et al., 2014). The researchers inserted a circularly permuted GFP variant between two transmembrane helices of a chicken voltage-sensitive domain such that the fluorescent protein is located extracellularly. Voltage-induced movements distort GFP and change its fluorescence. "ASAP1 is the first truly new design concept for voltage sensors in the last five years or so," says Lin.

Despite the different mechanisms of these sensors, their performance is comparable. Both sensors can report spike trains at higher speeds than those achieved by previously described sensors while still displaying easily detectable fluorescence changes. Both groups have tested their sensors in brain slices. The MacQ-mCitrine sensor can even report activity from dendrites in live mice. However, Schnitzer concedes that "the detection of action potentials of this type in live mice is really quite on the edge of the capability."

Voltage sensors have a bright future ahead. "In recent years, we have seen quite some substantial improvement in voltage sensor performance," says Lin. But both Lin and Schnitzer are trying to improve their sensors by increasing photostability, brightness and dynamic range. "The voltage sensors in general still have some improvements needed to really enable imaging with single-cell resolution in behaving mice, in the way it has become much more commonplace nowadays for calcium imaging," says Schnitzer.

It will be interesting to see how the performance of these new sensors compares for different applications. Schnitzer thinks that "it depends on the details of the application.... It would be reasonable for people to look at them both in the particular system of interest."

Nina Vogt

\section{RESEARCH PAPERS}

Gong, Y. et al. Imaging neural spiking in brain tissue using FRET-opsin protein voltage sensors. Nat. Commun. 5, 3674 (2014).

St-Pierre, F. et al. High-fidelity optical reporting of neuronal electrical activity with an ultrafast fluorescent voltage sensor. Nat. Neurosci. 17, 884-889 (2014).

The researchers designed only transcriptional activators, but Lu does not see the approach being limited to activators. "We have shown that it is pretty straightforward to swap domains fused to the Cas9 system and turn an activator into a repressor," he says.

Ron Weiss and his team, on the other hand, focused solely on CRISPR-based repressors "because," says Weiss, "they are the fundamental building blocks for complex logic circuits." Repressors can be combined into NAND (no output if both inputs are present) and NOR gates (output only if both inputs are absent), which in turn are sufficient to build any logic function.

Samira Kiani, a postdoc in the Weiss lab, was the driving force behind the repression devices (Kiani et al., 2014). Her approach to creating a pol II-driven gRNA was simpler than that of the Lu group. She expressed the gRNA directly downstream of a pol II promoter, from an intron, or from the $3^{\prime}$ untranslated region of a reporter gene and also tried different configurations of the gRNA target sites on the promoter to obtain high binding efficiency. Weiss recalls that the work involved a lot of optimization. "We definitely did not have functional devices at the first shot," he says.

Weiss's team created a layered three-promoter repressor cascade wherein one gRNA'dead' Cas9 (dCas9) complex blocks transcription of another gRNA that, in turn, when complexed with dCas9, blocks the expression of a reporter. Weiss plans to expand the current gRNA library and wants to create multi-input logic circuits that can, for example, distinguish cancer from noncancer cells.

Lu has a similar vision of developing circuits that can interface with natural networks to diagnose or treat a disease. Combining the pol II-driven gRNA designs of the two teams may lead to yet more efficient regulatory devices that help reach these goals faster.

Nicole Rusk

\section{RESEARCH PAPERS}

Kiani, S., et al. CRISPR transcriptional repression devices and layered circuits in mammalian cells. Nat. Methods 11, 723-726 (2014).

Nissim, L. et al. Multiplexed and programmable regulation of genetic networks with an integrated RNA and CRISPR/Cas toolkit in human cells. Mol. Cell 54, 698-710 (2014). 